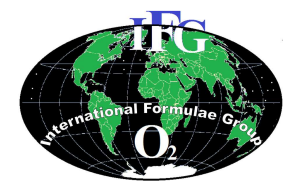

Available online at http://ajol.info/index.php/ijbcs

Int. J. Biol. Chem. Sci. 9(2): 944-953, April 2015

\title{
Comparaison des caractéristiques nutritionnelles et rhéologiques des bouillies infantiles préparées par les techniques de germination et de fermentation
}

\author{
Angèle KOUASSI KUNIMBOA ABRO AMOIN ${ }^{1 *}$, Edith ADOUKO AGBO ${ }^{1}$, \\ André GNAHE DAGO ${ }^{2}$, Albarin GRODJI GBOGOURI ${ }^{1}$, David KOUAKOU BROU ${ }^{1}$ et \\ GNAKRI DAGO ${ }^{2}$ \\ ${ }^{1}$ Université Nangui Abrogoua, Laboratoire de Nutrition et de Sécurité Alimentaire, \\ 02 BP 801 Abidjan 02, Côte d'Ivoire. \\ ${ }^{2}$ Université Jean Lorougnon Guédé de Daloa, BP 150 Daloa, Côte d'Ivoire. \\ *Auteur correspondant, E-mail: kunimboaangele@gmail.com
}

\section{RESUME}

Le sevrage est une étape importante dans l'alimentation du nourrisson. Aussi, les techniques de germination et de fermentation ont été utilisées pour améliorer les propriétés des farines composées à base de maïs, de sorgho et de soja. Les caractéristiques physicochimiques et rhéologiques, la composition nutritionnelle et la valeur énergétique des bouillies préparées à partir de ces farines ont été déterminées. Les résultats ont révélé, respectivement pour la farine composée germée et la farine composée fermentée, une teneur en glucides de $69,20 \%$ et $67,80 \%$, un taux de lipides de $7,5 \%$ et $4,5 \%$ et une teneur en protéines de $15,80 \%$ et $15,25 \%$. La valeur énergétique de la farine composée germée est de 402,3 kcal/100 g de matière sèche et celle de la farine composée fermentée est de $361,39 \mathrm{kcal} / 100 \mathrm{~g}$ de matière sèche. La bouillie issue de la farine composée germée a une matière sèche de $30,60 \%$, une densité énergétique de $122,4 \mathrm{kcal} / 100 \mathrm{~g}$ de matière sèche et une fluidité de $120 \mathrm{~mm} / 30 \mathrm{sec}$. La bouillie issue de la farine composée fermentée a une matière sèche de $30,05 \%$, une densité énergétique de $120,20 \mathrm{kcal} / 10 \mathrm{ml}$ et une fluidité de $100 \mathrm{~mm} / 30 \mathrm{sec}$. Ces farines composées fermentées et germées ont permis de préparer des bouillies infantiles conformes aux normes de l'OMS, pourraient contribuer à lutter contre la malnutrition infantile.

(C) 2015 International Formulae Group. All rights reserved.

Mots clés : Aliment de complément, nourrisson, malnutrition infantile, germination, fermentation.

\section{INTRODUCTION}

La malnutrition chez les enfants constitue un problème de santé publique dans le monde et particulièrement dans les pays en voie de développement (Tou, 2007). Elle apparaît généralement durant la période qui correspond à l'introduction de l'aliment de complément au lait maternel chez le nourrisson (Dewey et Brown, 2003). L'aliment de complément est un aliment donné aux nourrissons sous forme de bouillie à partir de l'âge de 6 mois (FAO, 2004). Il est spécialement conçu pour leur permettre de couvrir leurs besoins nutritionnels car après 6 mois, le lait maternel ne suffit plus à fournir entièrement les besoins nutritionnels du nourrisson en énergie et en protéines (OMS, 2003).

En Côte d'Ivoire, les aliments de complément sont, pour la plupart, préparés à 
partir des denrées locales, notamment les produits amylacés tels que les céréales et les tubercules. Toutefois, ces denrées ne subissent aucun traitement enzymatique préalable et entrent seuls dans la confection des bouillies sans adjonction d'aliments sources de protéines tels que les légumineuses ou le lait (Thomazic, 2003). Il s'en suit donc que pendant la cuisson, ces bouillies s'épaississent très rapidement. Compte tenu du petit volume d'estomac de l'enfant qui est de l'ordre de 30 $\mathrm{ml} / \mathrm{kg}$ de poids corporel (Sawadogo et al, 2003) en consomme très peu et semble être rassasié, mais manque de nutriments essentiels (Trèche, 2004). Pour pallier à cela, améliorer la densité énergétique et la fluidité des bouillies locales s'avère nécessaire. Ainsi, l'amidon des farines doit subir des traitements technologiques favorisant la rupture des liaisons glycosidiques afin de réduire le taux de gonflement et par conséquent augmenter leur fluidité et la densité énergétique à la cuisson. Ces traitements technologiques sont entre autres la fermentation et la germination (Mouquet et al., 2001 ; Mouquet et al., 2003). Il est donc opportun d'élaborer un aliment de complément répondant aux caractéristiques recherchées, c'est-à-dire, fluidité, densité énergétique et ayant une bonne valeur nutritionnelle. Notre étude consistera à faire une étude comparative des caractéristiques nutritionnelles et rhéologiques des bouillies préparées à base des produits locaux (maïs, sorgho, soja) selon les techniques de fermentation et de germination.

\section{MATERIEL ET METHODES}

\section{Echantillonnage}

Les farines composées ont été élaborées à partir de grains de maïs (Zea mays), de sorgho blanc (Guinea sorghum) et de soja (Glycine max). Un kilogramme de grains de maïs, sorgho et de soja ont été achetés au « Forum des marchés » d'Adjamé à Abidjan Côte d'Ivoire. Le choix de ces céréales se justifie par le fait qu'ils sont disponibles en toute saison.

Pour la farine composée fermentée, le ferment utilisé a été le ferment de manioc appelé localement «Magnan ». Le manioc a été acheté au «Forum des marchés » d'Adjamé et $500 \mathrm{~g}$ de manioc frais ont été épluchés et braisés à feu de charbon pendant 10 minutes. Ils ont été ensuite refroidis à la température ambiante $28 \pm 1{ }^{\circ} \mathrm{C}$ puis emballés dans un sachet en plastique. L'ensemble a été placé dans un panier puis conservé à la température du magasin de stockage $30 \pm 2{ }^{\circ} \mathrm{C}$. Cependant 3 jours à l'abri de l'air et de la lumière. Au terme de cette conservation, le levain a été ainsi obtenu.

\section{Production des farines}

Les grains ont été triés, vannés et lavés au préalable avant la production des farines.

\section{Préparation de farine composée germée}

Les grains de maïs, de sorgho et de soja ont été trempés séparément dans un récipient ouvert pendant $48 \mathrm{~h}$ puis étalés sur un tissu en coton humidifié pendant 3 jours pour la germination. Ensuite les grains qui ont effectivement germés ont été séchés à $65^{\circ} \mathrm{C}$ à l'étuve pendant $48 \mathrm{~h}$ puis débarrassés de leurs plantules avant d'être finement broyés avec un broyeur de type Forplex et tamisés avec un tamis de diamètre $500 \mu \mathrm{m}$. Le conditionnement et la conservation des farines s'est fait séparément.

Préparation de la farine composée fermentée

Les grains de maïs, de sorgho et de soja ont été broyés séparément et tamisés avec un tamis de diamètre $500 \mu \mathrm{m}$. Les différents grains n'ont pas été trempés au préalable. La farine composée fermentée a été élaborée en mélangeant, dans des proportions variées, les précédentes farines, le ferment de manioc et le saccharose dans $100 \mathrm{ml}$ d'eau. Le mélange a été mis dans un sachet plastique transparent. Après avoir bien entassé la pâte pour permettre la fermentation en anaérobiose, le sachet a été fermée hermétiquement et placé dans un récipient en plastique. La fermentation a duré $72 \mathrm{~h}$ (Brou, 2000).

\section{Formulation d'aliments de sevrage Formulation de la farine composée germée} Les formulations $\mathrm{F}_{1}$ à $\mathrm{F}_{3}$ ont été élaborées à partir des farines de maïs, de sorgho et de soja germées (Tableau 1). 
La formule $\mathrm{F}_{1}$ désigne le mélange de la farine de maïs germé $(50 \%)$ et de sorgho germé $(50 \%)$ sans incorporation de soja germé et de sucre .Ensuite la formule $\mathrm{F}_{2}$ indique le mélange de farine de maïs germé et de sorgho germé dans les proportions respectives $33,34 \%$; $33,34 \%$ avec $33,34 \%$ d'incorporation de la farine de soja germé. Enfin la formule $F_{3}$ désigne le mélange de farine de maïs germé, de sorgho germé et de sucre dans les proportions respectives de $29,40 \%$; $29,40 \%$ et $11,80 \%$ avec $29,40 \%$ d'incorporation de la farine de soja.

\section{Formulation de la farine composée}

\section{fermentée}

Les formulations $\mathrm{F}_{\mathrm{A}}$ et $\mathrm{F}_{\mathrm{B}}$ ont été réalisées à partir des farines de maïs, de sorgho et de soja fermentés (Tableau 2).

La formule $\mathrm{F}_{\mathrm{A}}$ désigne le mélange de farines de maïs, de sorgho, de soja, de ferment et de sucre dans les proportions respectives de $28,30 \% ; 28,30 \% ; 28,30 \% ; 3,8 \%$ et $11,30 \%$ sans incorporation du maïs germé. La formule $F_{B}$ indique le mélange de farines de maïs, de sorgho, de soja, de ferment et de sucre dans les proportions respectives de $26,32 \%$; $26,32 \% ; 26,32 \% ; 7 \%$ et $10,53 \%$ avec $3,51 \%$ d'incorporation du maïs germé.

\section{Analyses biologiques}

Les analyses effectuées ont portées sur la matière sèche, le dosage des protéines et l'extraction de lipides par la méthode AOAC (1995). Le nombre total d'hydrates de carbone et les sucres totaux ont été déterminés selon la méthode de Bertrand et Thomas (1910). La teneur en amidon a été déduite en calculant la différence entre la teneur totale en hydrate de carbone et la teneur en sucre totaux. Le dosage de la cellulose a été réalisé selon la méthode de Prosky et al. (1988). Le phosphore a été dosé selon la méthode de Briggs (1977) et le fer selon la méthode colorimètre d'orthophénanthroline (Audigie et al., 1978). Le magnésium, le potassium, le sodium, le calcium, le zinc, le cuivre et le manganèse ont été déterminé par spectrométrie d'absorption atomique de type Perkin-Elmer, modèle 1100 (BIPEA, 1976).

La consistance de la bouillie a été appréciée à travers sa vitesse d'écoulement qui a été évaluée par rapport de la distance parcourue par $100 \mathrm{ml}$ de bouillies pendant 30 secondes et mesurée à l'aide du consistomètre de Bostwick selon la méthode de Vieu et al. (2001). A partir de la matière sèche de la bouillie la densité d'énergie (DE) a été calculée selon l'équation d'Atwater et Benedict (1902).

$\mathrm{DE}=(9 \times$ lipides $(\%)+4 \mathrm{x}$ protéines $(\%)+4 \times$ glucides $(\%)) \times$ MS (\%).

\section{Analyses statistiques}

Les analyses statistiques des données ont été effectuées à l'aide des logiciels STATISTICA 7.1 (StatsoftInc, Tulsa-USA Headquarters) et XLSTAT-Pro 7.5.2 (Addinsoft Sarl, Paris-France). Les comparaisons entre les variables dépendantes ont été déterminées au moyen de l'analyse de variance (ANOVA) à deux facteurs et du test de Duncan. La signification statistique a été définie à $\mathrm{p} \leq 0,05$.

\section{RESULTATS}

Formulation des farines composées par le procédé de germination

L'appréciation des caractéristiques physicochimiques des bouillies a été faite à partir de la détermination de la fluidité, de la matière sèche, de la teneur en protéines et de la densité énergétique. Ainsi ces paramètres ont été déterminés pour toutes les formulations de farines composées $\left(\mathrm{F}_{1}, \mathrm{~F}_{2}\right.$ et $\mathrm{F}_{3}$ ) (Tableau 3).

A $30 \mathrm{~g} \mathrm{du}$ poids de mélange, la formulation $\mathrm{F}_{1}$ (à base de maïs germé et de sorgho germé dans les proportions 50/50) présente un taux de matière sèche de la bouillie égal à 26,50\%, une fluidité de 130 $\mathrm{mm} / 30 \mathrm{sec}$, un taux de protéines de $13,50 \%$ et une densité énergétique de $106 \mathrm{kcal} / 100 \mathrm{ml}$. Cependant, ces taux de matière sèche et de protéines sont inférieurs respectivement à 
$30 \%$ de matière sèche et $15 \%$ de protéines (valeurs recommandées par l'OMS) et ont été améliorés par l'incorporation de farine de soja germé, soit la formulation $\mathrm{F}_{2}$. Toutefois, la bouillie issue de cette formulation présente une teneur suffisante en protéine de $15,80 \%$ mais a une matière sèche de $28,15 \%$ qui demeure insuffisante. La fluidité est de 125 $\mathrm{mm} / 30 \mathrm{sec}$. La densité énergétique est de $112,6 \mathrm{kcal} / 100 \mathrm{ml}$ de bouillie. A cette étape l'amélioration des paramètres a été faite par l'ajout de saccharose. Ainsi, en apportant $11,80 \%$ de saccharose à la formulation $\mathrm{F}_{2}$, on obtient la formulation $\mathrm{F}_{3}$ qui améliore le goût de la bouillie, de même que le taux de matière sèche qui passe de $28,15 \%$ à $30,60 \%$. La densité énergétique augmente de 112,6 kcal à $122,4 \mathrm{kcal} / 100 \mathrm{ml}$ de bouillies et la fluidité passe de $125 \mathrm{~mm} / 30 \mathrm{sec}$ à $120 \mathrm{~mm} / 30 \mathrm{sec}$. Toutefois, le taux de protéines demeure égal à $15,80 \%$.

De toutes les formulations étudiées, seule la farine $\mathrm{F}_{3}$ qui renferme $29,40 \%$ de maïs germé, $29,40 \%$ de sorgho germé, $29,40 \%$ de soja germé et $11,80 \%$ de sucre a permis de préparer une bouillie dont la densité énergétique était de 122,4 kcal/100 ml de bouillies et pouvant couvrir les besoins énergétiques du nourrisson. La matière sèche, la teneur en protéines et la fluidité qui ont été respectivement de $30,60 \%, 15,80 \%$ et 120 $\mathrm{mm} / 30$ sec sont conformes aux recommandations de l'OMS.

\section{Formulation de la farine composée par le procédé de fermentation}

Les caractéristiques physicochimiques (fluidité, matière sèche, teneur en protéines et densité énergétique) des bouillies élaborées selon le procédé de fermentation ont été énumérées dans le Tableau 4. La farine composée fermentée (formulation $\mathrm{F}_{\mathrm{A}}$ ), dont la composition renferme $28,30 \%$ respectivement de farine de maïs, de sorgho, de soja, $3,80 \%$ de ferment de manioc et de $11,30 \%$ de saccharose, a permis d'obtenir une bouillie de fluidité de $90 \mathrm{~mm} / 30 \mathrm{sec}$ avec un taux de protéine de 15,17\% acceptables selon les normes de l'OMS (2003). Cependant, la matière sèche qui est de $22,67 \%$ et la densité énergétique de 90,68 kcal/100 $\mathrm{ml}$ de bouillies sont insuffisantes. L'amélioration de la matière sèche et de la densité énergétique a été faite par l'incorporation de 3,51\% de maïs germé à la formulation $\mathrm{F}_{\mathrm{A}}$. Soit la farine de formulation $\mathrm{F}_{\mathrm{B}}$ dont la matière sèche est de $30,05 \%$, la densité énergétique de 120,20 $\mathrm{kcal} / 100 \mathrm{ml}$ de bouillies, le taux de protéine de $15,17 \%$ et la fluidité de $100 \mathrm{~mm} / 30 \mathrm{sec}$.

\section{Composition nutritionnelle des farines composées $F_{B}$ et $F_{3}$}

A part le taux de lipides et la valeur énergétique, les teneurs en glucides, protéines, cellulose, cendres et amidon des farines composées germées et fermentées ne diffèrent pas significativement entre elles (Tableau 5). La farine composée germée $\mathrm{F}_{3}$ contient $69,20 \pm 0,8 \%$ de glucides, $15,80 \pm 1,4 \%$ de protéines et $7,5 \pm 0,5 \%$ de lipides pour une valeur énergétique qui est de 402,37 kcals, tandis que la farine composée fermentée contient $67,80 \pm 0,3 \%$ de glucides, $15,25 \pm 0,83 \%$ de protéines et $4,5 \pm 0,5 \%$ de lipides avec une valeur énergétique de 361,39 kcals. La teneur en cellulose est de $4 \pm 1 \%$ dans la farine composée germée et de $3,2 \pm 0,1 \%$ dans la farine fermentée. Les teneurs en cendres sont de $2,8 \pm 0,5 \%$ et $2,6 \pm 0,4 \%$ respectivement dans la farine composée germée et la farine composée fermentée. Il n'y a pas de différence significative au seuil de $p \geq 0,05$ de la valeur énergétique de la farine composée germée et de la farine standard.

Les teneurs en minéraux de la farine composée germée diffèrent statistiquement de ceux de la farine composée fermentée (Tableau 6). Ainsi, les teneurs en potassium et en calcium sont plus élevées dans la farine composée germée que dans la farine composée fermentée avec respectivement $383,79 \pm 1,06 \mathrm{mg}$ de potassium, 300,5 $\pm 1,5$ $\mathrm{mg}$ de calcium contre $289,4 \pm 1,5 \mathrm{mg}$ de 
potassium et $268,5 \pm 1,83 \mathrm{mg}$ de calcium. Par contre, a teneur en phosphore $(170 \pm 2,30 \mathrm{mg})$ de la farine composée fermentée est plus élevée que celle de la farine composée germée $(154 \pm 1 \mathrm{mg})$. Il en est de même pour les teneurs en sodium, soit $46 \pm 1,2 \mathrm{mg}$ pour la $\mathrm{F}_{\mathrm{B}}$ et $40 \pm 1 \mathrm{mg}$ pour la $\mathrm{F}_{3}$. Les teneurs en fer et en zinc sont plus élevées dans la farine composée germée $(13,5 \pm 0,5 \mathrm{mg}$ et $10,03 \pm$ $0,97 \mathrm{mg}$ respectivement) que dans la farine composée fermentée $(12,58 \pm 0,5 \mathrm{mg}$ et $7,5 \pm$ $0,5 \mathrm{mg}$ respectivement).

Tableau 1 : Formulation d'aliments de complément par la technique de germination.

\begin{tabular}{lcccc}
\hline \multicolumn{5}{c}{ Ingrédients $(\%)$} \\
\hline Formules & Maïs germé & Sorgho germé & Soja germé & Sucre \\
\hline $\mathrm{F}_{1}$ & 50 & 50 & 0 & 0 \\
$\mathrm{~F}_{2}$ & 33,34 & 33,34 & 33,34 & 0 \\
$\mathrm{~F}_{3}$ & 29,40 & 29,40 & 29,40 & 11,80 \\
\hline
\end{tabular}

Tableau 2 : Formulation d'aliments de complément par la technique de fermentation.

\begin{tabular}{lllllll}
\hline & & & Ingrédients & $(\%)$ & \\
\hline Formules & Maïs & Sorgho & Soja & Maïs germée & Sucre & Ferment \\
$\mathrm{F}_{\mathrm{A}}$ & 28,30 & 28,30 & 28,30 & 0 & 11,30 & 3,8 \\
$\mathrm{~F}_{\mathrm{B}}$ & 26,32 & 26,32 & 26,32 & 3,51 & 10,53 & 7 \\
\hline
\end{tabular}

Tableau 3 : Caractéristiques physico-chimiques de bouillies préparées à partir du mélange de maïs germé et de sorgho germé.

\begin{tabular}{cccccc}
\hline Formules & Proportion $(\boldsymbol{\%})$ & Fluidité & \multicolumn{2}{c}{ Caractéristiques des bouillies } \\
\hline & & $\begin{array}{c}\text { Matière } \\
\text { mm/30 sec }\end{array}$ & $\begin{array}{c}\text { Protéche }(\boldsymbol{\%}) \\
(\boldsymbol{\%})\end{array}$ & $\begin{array}{c}\text { Densité Energétique } \\
(\mathbf{k c a l} / \mathbf{1 0 0} \mathbf{~ m l})\end{array}$ \\
\hline F1 & $50 / 50$ & 130 & 26,50 & 13,50 & 106 \\
F2 & $3433,34 / 33,34 / 33,34$ & 125 & 28,15 & 15,80 & 112,6 \\
F3 & $29,40 / 29,40 / 29,4 / 11,80$ & 120 & 30,6 & 15,80 & 122,4 \\
\hline
\end{tabular}

Tableau 4: Caractéristiques physico-chimiques des bouillies obtenues par incorporation du ferment au mélange de farines de maïs, de sorgho et de soja.

\begin{tabular}{lcccccc}
\hline Formules & $\begin{array}{c}\text { Proportions } \\
(\boldsymbol{\%})\end{array}$ & $\begin{array}{c}\text { Taux de } \\
\text { ferment }(\boldsymbol{\%})\end{array}$ & & \multicolumn{2}{c}{ Caractéristiques des bouillies fermentées } \\
& & & $\begin{array}{c}\text { Fluidité } \\
\mathbf{m m} / \mathbf{3 0 s e c}\end{array}$ & $\begin{array}{c}\text { Matière } \\
\text { sèche }(\boldsymbol{\%})\end{array}$ & $\begin{array}{c}\text { Protéine } \\
(\boldsymbol{\%})\end{array}$ & $\begin{array}{c}\text { Densité Energétique } \\
(\mathbf{K c a l} / \mathbf{1 0 0} \mathbf{~ m l})\end{array}$ \\
\hline $\mathrm{F}_{\mathrm{A}}$ & $28,30 / 28,30 / 28,30$ & 3,80 & 90 & 22,67 & 15,17 & 90,68 \\
$\mathrm{~F}_{\mathrm{B}}$ & $26,32 / 26,32 / 26,32$ & 7 & 100 & 30,05 & 15,25 & 120,20 \\
\hline
\end{tabular}


Tableau 5 : Composition chimique de la farine composée germée $\mathrm{F}_{3}$ et farine composée fermentée $\mathrm{F}_{\mathrm{B}}$.

\begin{tabular}{lccc}
\hline $\begin{array}{l}\text { Nutriments } \\
\text { (g/100 g de farines) }\end{array}$ & $\begin{array}{c}\text { Farine composée } \\
\text { Germée } \mathbf{F}_{\mathbf{3}}\end{array}$ & $\begin{array}{c}\text { Farine composée } \\
\text { fermentée } \mathbf{F}_{\mathbf{B}}\end{array}$ & $\begin{array}{c}\text { Farine Standard } \\
\text { FAO/OMS (2006) }\end{array}$ \\
\cline { 2 - 4 } & $95 \pm 1^{\mathrm{a}}$ & $94 \pm 1,5^{\mathrm{a}}$ & 95 \\
Matière sèche & $69,20 \pm 0,8^{\mathrm{a}}$ & $67,80 \pm 0,3^{\mathrm{a}}$ & 68 \\
Glucides & $61,5 \pm 0,5^{\mathrm{a}}$ & $63,5 \pm 0,2^{\mathrm{a}}$ & 64 \\
Amidon & $7,5 \pm 0,5^{\mathrm{b}}$ & $4,5 \pm 0,5^{\mathrm{a}}$ & 8 \\
Lipides & $15,80 \pm 1,4^{\mathrm{a}}$ & $15,25 \pm 0,83^{\mathrm{a}}$ & 15 \\
protéines & $4 \pm 1^{\mathrm{a}}$ & $3,5 \pm 0,1^{\mathrm{a}}$ & 3,8 \\
Cellulose & $2,8 \pm 0,5^{\mathrm{a}}$ & $2,6 \pm 0,4^{\mathrm{a}}$ & 2,9 \\
Cendres & $402,37 \pm 2,63^{\mathrm{b}}$ & $361,39 \pm 1,52^{\mathrm{a}}$ & 400 \\
\hline Valeur énergétique & \multicolumn{2}{c}{ Les chiffres suivis du même exposant dans une rangée ne diffèrent pas significativement au seuil $\mathrm{P}>0,05}$.
\end{tabular}

Tableau 6: Teneurs en minéraux des farines composées.

\begin{tabular}{cccc}
\hline $\begin{array}{c}\text { Minéraux } \\
(\mathbf{m g} / \mathbf{1 0 0 g} \text { farines })\end{array}$ & $\begin{array}{c}\text { Farine composée } \\
\text { germée } \mathbf{F}_{\mathbf{3}}(\mathbf{g})\end{array}$ & $\begin{array}{c}\text { Farine composée } \\
\text { fermentée } \mathbf{F}_{\mathbf{B}}(\mathbf{g})\end{array}$ & $\begin{array}{c}\text { Composition souhaitable } \\
(\mathbf{m g} / \mathbf{1 0 0 g}) \mathbf{F A O} / \mathbf{O M S} \\
\mathbf{( 2 0 0 6 )}\end{array}$ \\
\hline Potassium & $383,79 \pm 1,06^{\mathrm{b}}$ & $289,4 \pm 1,50^{\mathrm{a}}$ & 408,7 \\
Calcium & $300,5 \pm 1,5^{\mathrm{b}}$ & $268,5 \pm 1,83^{\mathrm{a}}$ & 341,2 \\
Sodium & $40 \pm 1^{\mathrm{a}}$ & $46 \pm 1,20^{\mathrm{b}}$ & 60 \\
Phosphore & $154 \pm 1^{\mathrm{a}}$ & $170 \pm 2,30^{\mathrm{b}}$ & 281,2 \\
Fer & $13,5 \pm 0,50^{\mathrm{b}}$ & $12,58 \pm 0,50^{\mathrm{a}}$ & 8,5 \\
Magnésium & $85,75 \pm 0,25^{\mathrm{b}}$ & $74,3 \pm 0,66^{\mathrm{a}}$ & 48,7 \\
zinc & $10,03 \pm 0,97^{\mathrm{b}}$ & $7,5 \pm 0,50^{\mathrm{a}}$ & 3,7 \\
Cuivre & $2,87 \pm 0,02^{\mathrm{b}}$ & $1,8 \pm 0,14^{\mathrm{a}}$ & 0,1 \\
\hline
\end{tabular}

En ligne les chiffres qui ne sont pas suivis de la même lettre diffèrent significativement au seuil de $p \leq 0,05$.

\section{DISCUSSION}

Les caractéristiques physico-chimiques des bouillies élaborées ont révélé que la teneur en matière sèche de la farine est corrélée à la fluidité. En effet, plus la teneur en matière sèche est faible, plus la bouillie est fluide et moins dense énergétiquement. Pour les nourrissons, de telles bouillies sont sources de carences protéino-énergétiques susceptibles de favoriser une perte de poids. Au niveau de la farine composée germée, l'incorporation de la farine de soja et du saccharose a amélioré le goût de la bouillie et a permis au taux de matière sèche dépasser de $26,80 \%$ à $30,60 \%$, la densité énergétique d'augmenter de 107,4\% à 122,4 kcals, au taux de protéines de passer de $13,50 \%$ à $15,80 \%$ et à la fluidité de baisser de 130 à $120 \mathrm{~mm} / 30 \mathrm{sec}$. Ces résultats correspondent à ceux de Zannou et al. (2011) qui ont montré que l'étude de la valeur nutritionnelle des farines élaborées FAS (Attiéké et Soja) et FMS (Manioc et Soja) donne pour FAS les taux de 13\% de protéines, $10 \%$ de lipide et $63 \%$ de glucides avec une valeur énergétique de $394 \mathrm{kcals}$. En ce qui concerne la farine FMS les taux de $14 \%$ de protéine, $10 \%$ de lipide et $61 \%$ de glucides avec une valeur énergétique de 390 kcals.

L'avantage de l'incorporation de soja dans les farines germée et fermentée se 
justifierait par le fait qu'il renferme des protéines de bonnes valeurs biologiques contenant tous les acides aminés essentiels ainsi que des vitamines et des minéraux. La teneur importante en lipides du soja lui confère un bon pouvoir calorifique. Par ailleurs, la consommation des aliments sources de protéines végétales (les légumineuses) est à encourager car elles sont disponibles et peu coûteuses par rapport aux protéines animales. En outre, la supplémentation des céréales par le soja a favorisé l'augmentation de la teneur en protéines tant dans la farine composée germée $(15,80 \%)$ que dans la farine composée fermentée $(15,25 \%)$ et ces teneurs sont supérieures à celle de la farine standard (15\%). Ces résultats sont en accord avec ceux de Solomon (2005) et Onilude (2009) qui ont révélé dans leurs études que la teneur en protéine des céréales et des légumineuses combinées est meilleure que celle produite à partir de céréales seules. La germination et la fermentation ont par ailleurs amélioré considérablement la teneur et la digestibilité des protéines.

Le taux d'humidité des différentes farines composées (5 à 5,5\%) est appréciable, la norme indiquant un taux de $5 \%$ dans les farines infantiles. De plus, la faible teneur en humidité des farines augmente leur durée de conservation. Ces résultats sont similaires à ceux de Alozié et al. (2009) qui ont déterminé dans la farine de bambara un taux d'humidité de $5 \%$, des teneurs en glucides et en protéines comprises entre 60 et $70 \%$ pour les glucides et entre 12 et $15 \%$ pour les protéines. Ces valeurs sont conformes à celles de la norme (OMS/UNICEF, 2003) et de Mouquet et al. (2008). De plus, la farine composée germée renferme un taux de lipide $(7,5 \%)$ et une valeur énergétique (402,37 kcals) sensiblement égaux à ceux de la norme. Cependant, la valeur énergétique $(361,39$ kcals) et la teneur en lipide $(4,5 \%)$ de la farine composée fermentée sont inférieures à celles de la farine standard $(7 \%)$ de lipides pour une valeur énergétique de $400 \mathrm{kcals}$ ) et par là, à ceux de la farine composée germée. Cela pourrait signifier que la germination améliorerait mieux la valeur nutritive des aliments que la fermentation (Syed et al., 2011).

L'incorporation de la farine germée à la bouillie fermentée a permis de préparer des bouilles ayant une consistance de $100 \mathrm{~mm} / 30$ sec, une matière sèche de $30,05 \%$ et une densité énergétique de 120,20 kcal/100 ml. Ces résultats sont en accord avec ceux de Elenga et al. (2012) qui ont montré que l'incorporation de malt et de carbonate de calcium à la pâte fermentée de maïs-arachide a permis de préparer des bouillies ayant une consistance recommandée de $120 \mathrm{~mm} / 30 \mathrm{sec}$ pour teneur en matière sèche de $20 \mathrm{~g}$ pour $100 \mathrm{~g}$ de bouillies.

Les teneurs en potassium, calcium, magnésium, fer, zinc et cuivre sont plus élevées dans la farine composée germée que dans la farine composée fermentée. Cela est dû au fait que la germination augmenterait la rétention des minéraux (Egli, 2001; Helland et al., 2002). La présence de ces minéraux dans les farines composées est bénéfique car ils interviennent dans le développement de l'organisme et dans la croissance de l'enfant et pourraient limiter l'apparition de carences nutritionnelles. Ainsi, le fer intervient dans la constitution de l'hémoglobine, de la myoglobine et de nombreuses enzymes. Il est aussi indispensable pour un grand nombre de réactions métaboliques (Lokombé et al., 2004) tout comme le sodium qui permet à l'organisme de réguler les entrées et les sorties de potassium afin d'en réduire fortement le risque de carence. Le calcium et le phosphore assurent quant à eux la rigidité des os et favorisent la croissance des enfants (Weaver et al., 2007). Le zinc représente avec le fer l'un des minéraux les plus concentrés au 
niveau du cerveau. Aussi, il est indispensable de couvrir les besoins en zinc chez le nourrisson où la croissance cérébrale est encore importante. Le zinc est aussi impliqué dans l'immunité car il réduirait l'incidence et la gravité des diarrhées chez l'enfant (Lokombé et al., 2004). Le cuivre (en très faible quantité dans le corps humain) entre dans la composition de nombreux enzymes et intervient également dans le phénomène de minéralisation osseuse, de renforcement de l'immunité et dans les métabolismes du fer et du glucose (Lokombé et al., 2004). Le magnésium quant à lui est nécessaire pour les réactions biochimiques dans le corps, permettant de maintenir le muscle, améliorant le fonctionnement du nerf, le maintien du rythme cardiaque et la régulation du taux de sucre dans le sang (Saris et al., 2000).

Les méthodes traditionnelles comme la germination et la fermentation ont tendance à améliorer la qualité des aliments en nutriments, ces résultats sont en accords avec ceux de Brou (2000).

\section{Conclusion}

Les études réalisées en vue d'apporter notre contribution à l'amélioration de l'aliment de complément du nourrisson en période de sevrage ont permis de mettre au point des farines infantiles: la farine composée germée $\mathrm{F}_{3}$ et la farine composée fermentée $F_{B}$. Ces deux farines composées renferment de bonnes teneurs en protéines, en lipides, en glucides et en minéraux, selon les recommandations de la FAO/OMS. Cependant, ces taux sont plus élevés dans la farine composée germée que dans la farine composée fermentée. Le procédé de germination améliorerait donc mieux la qualité des farines que la fermentation. Par ailleurs, la supplémentation de la farine fermentée par la farine germée a permis d'améliorer la consistance des bouilles et la densité énergétique, augmentant ainsi pour les nourrissons et les jeunes enfants le potentiel de surmonter la malnutrition protéinoénergétique. En perspective nous envisageons à évaluer sur les animaux afin d'apprécier la qualité des farines composées in vivo ainsi que leurs effets secondaires.

\section{REFERENCES}

AOAC. 1995. Méthodes d'Analyses Officielles (16 ème édn). Association of Official Analytical Chemists; 250 p.

Alozie YE, Iyam MA, lawalo O, udofia U,Ani IF. 2009. Utilisation de la farine de Bambara comme aliment de complément. Londres. Journal of Food Technology, 7(4): 111-114.

Atwater WO, Benedict FG. 1902. Experiments on the metabolism of energy in the human body, 1898-1900. United States. Office of Experiment Stations, bulletin $\mathrm{N}^{\circ} 109$. Government Printing Office: Washington, DC.

Brou K, Camara F, N'Dri Y, Akaffou NC, Djeni NT. 2008. Effect of two mills techniques and flours particles size on some physicochemical properties of millet flour. Journal of Food Technology, 6(6): 231-236.

Brou K. 2000. La cofermentation comme stratégie pour l'amélioration de la valeur nutritionnelle des aliments de complément dans les pays en développement. Thèse de doctorat 3emè cycle. Université de Cocody (Côte d'Ivoire), p.126.

Bertrand G, Thomas P. 1910. Guide pour les Manipulations de Chimie Biologie. Dund : Paris.

BIPEA. 1976. Recueil des méthodes d'analyses des communautés européennes. Bureau interprofessionnel d'études analytiques: Gennevilliers. France ; 140.

Dewey KG, Brown KH. 2003. Undated on technical issues concerning 
complementary feeding of young children in developing countries and applications for intervention programs. Food Nutr. Bull, 24(1): 5-28.

Elenga M. 2012.Amélioration de la qualité nutritionnelle et de la densité énergétique des bouilles de maïs fermenté et de la farine d'igname utilisée comme aliment de complément du nourrisson. Thèse de Doctorat de l'Université de Marien Ngouabi, 104p.

Egli M. 2001. Les méthodes traditionnelles de transformation des aliments de sevrage. Thèse de doctorat. Fédéral Suisse, Techn. Zurich, 130p.

FAO/OMS. 2009. Programme mixte FAO/OMS sur les normes alimentaires. Commission du Codex Alimentarius, $32^{\text {ème }}$ session Rome (Italie), 29 juin- 4 juillet 2009. Rapport de la $30^{\text {ème }}$ session du comité du codex sur la nutrition et les aliments diététiques ou de régime. Le Cap (Afrique du Sud) 3-7 Novembre 2008, 1$223 \mathrm{p}$.

FAO/OMS. 2006. Programme mixte FAO/OMS sur les normes alimentaires. Rapport des vingt -septième sessions du comité du codex sur la nutrition et les aliments diététiques ou de régime. ALINOM 06/29/26, 105 p.

FAO. 2004. Undernourishment around the world. In: The state of food insecurity in the world 2004. The Organization, 2004, Rome:

Helland MH, Wickland T. 2002. Effet de temps de germination sur l'alpha amylase et la viscosité de la bouillie de maïs. Res Alimentaire Int., 35: 315-321.

Weaver CM, Mobley SL. 2007. Calcium intake,body fat,and bones a complex relation. Am. J. Clin. Nutr., 86(3): 527.

Lokombé-Léké A, Mullié C. 2004. Nutrition $\mathrm{du}$ nourrisson et diversification alimentaire. Cahiers de Nutrition et Diététique, 39: 349-359.
Kaushick G, Satya S, Naik SN. 2010. Effet de la production technique de traitement sur la qualité nutritionnelle du Soja. Mditerr. J. Nutri. Metab., 3(1): 39-46.

Mouquet C, Icard C, Guyot JP, Tou EH, Rochette I, Trèche S. 2008. Consumption patter,biochemical composition and nutritional value of fermented pearl millet gruels in Burkina Faso, International, Journal of Food Sciences and Nutrition, 59(7): 716-729.

Mouquet C, Salvignol B, Van Hoan N, Monvois J, Trèche S. 2003. Ability of a very low cost extruder to produce Instant flours at a small-scale level in Vietnam. Food Chemistry, 82: 249-55.

Mouquet C, Trèche S. 2001. Viscosity of gruels for infant a comparaison of measurement procedures. Int J Food Sci Nutr, 52: 389-400.

Onilude AA. 2009. Microbiological and chemical changes during production of malted and fermented Cereal- legume weaning foods. Advances in Food Sciences, 31(3):139-145.

OMS/UNICEF. 2003. Alimentation complémentaire des jeunes enfants dans les pays en développement. OMS: Genève; 130-131.

Solomon M. 2005. Nutritive value of three potential complementary goods based on cereals and legumes. Agriculture Nutritive, 5(2):1-14.

Syed AS, Aurang Z, Tariq M, Nadia N, Muhammad S, Abdul A, Asim M. 2011. Effets de la germination sur la qualité nutritionnelle des variétés de haricot mungo. Revue Africaine de la Recherche Agricole, 6(22): 5091-5098.

Sawadogo PS, Prevel MY, Savy M, Kameli Y, Traore AS. 2003. Pratiques d'alimentation du nourrisson en zone rurale au Burkina Faso: description et conséquences nutritionnelles. $2^{\text {ème }}$ Atelier International sur les voies alimentaires 
d'amélioration des situations nutritionnelles, Ouagadougou, 23-28 Novembre 2003.

Saris NE, Mervaala E, Karppanen H, Khawaja JA, Lewenstam A. 2000. Magnésium: an update on physiological, clinical, and analytical aspects. Clinica Chimica Acta, 294: 1-26.

Thomazic M. 2003. Caractérisation de la commercialisation et de la vente de potopoto. Mémoire de DESS, Université de Montpellier II.

Tou EH, Mouquet C, Rochette I, Traore AS, Trèche S, Guyot JP. 2007. Effect of three different process combinations (cooking, addition of malt and inoculation by back slopping) on the fermentation kinetics microflora and energy density of "ben saalga", a pearl millet based fermented gruel from Burkina Faso. Food Chemistry, 100: 935-945.

Trèche S. 2004. Les bouillies fluides, bébés bien nourris. In: Information pour le développement agricole des pays ACP, $\mathrm{N}^{\circ} 110$, Avril 2004.

Vieu MC, Traore TS. 2001. Effects of energy density and sweetness of gruels on Burkinabe infant energy intakes in free living condition. J. Food Sci. Nutr., 52: 213-218.

Zannou-Tchoko V, Ahui-Bitty L,Kouame K, Bouaffou K, Dally T. 2011.Utilisation de la farine de maïs germée source d'alpha amylase pour augmenter la densité énergétique des bouillie de sevrage à base de manioc et de son dérivé, l'attiéké. Journal of Applied Biosciences, 37: 24772484. 